\title{
Poly(1-alkyne)s as a Comb-Like Polymer with a Rigid Main-Chain
}

\author{
Kenji Yokota, ${ }^{*}$ Masao Ohtubo, Tadamichi Hirabayashi, \\ and Yoshihito INAI \\ Department of Materials Science and Engineering, \\ Nagoya Institute of Technology, Gokiso-cho, Showa-ku, \\ Nagoya 466, Japan
}

(Received March 25, 1993)

\begin{abstract}
Alkynes of even carbon numbers from 6 to 18 were polymerized by using W, $\mathrm{Mo}$, and Fe catalysts to give poly(1-alkynes), a type of comb-like polymer with a rigid conjugated polyene main-chain.$$
\begin{aligned}
\mathfrak{C H}= & \mathrm{C}\} \\
& \left(\stackrel{\mathrm{C}}{\mathrm{C}} \mathrm{H}_{2}\right)_{n} \mathrm{H} \quad n=4 \text { to } 16 .
\end{aligned}
$$

The extents of their side-chain crystallization were determined by differential scanning calorimetry and discussed in comparison with poly(1-alkene) comb-like polymers which have a flexible polymethylene main chain. Poly(1-alkynes) showed lower extents of side-chain crystallization than poly(1-alkenes) of the same carbon numbers because of their rigid main-chain structure.

KEY WORDS 1-Alkyne / Poly(1-alkyne)/Comb-Like Polymer / Metathesis Polymerization / Crystallization /
\end{abstract}

In previous papers, ${ }^{1-4}$ we synthesized the following widely spaced comb-like polymers $1-4$.<smiles>[R]OC(=O)C(C)CCC=CCC</smiles><smiles>[R]OC(=O)C(C)(C)CCCCCC</smiles><smiles>[R]C(CO)COC(=O)CCC(C)=O</smiles>

* To whom correspondence should be addressed.
Here $\mathrm{R}$ denotes long alkyl groups with $12-18$ even carbon numbers and $\mathrm{X}$ denotes a hydrogen or methyl group. The alkyl sidechains of these widely spaced comb-like polymers crystallized as those of conventional vinyl type comb-like polymers in spite of their wide spaces. The extent of side-chain crystallization as given in the $N$ number, which was estimated from the thermal analysis data to show the methylene carbon numbers involved in the crystallization, was influenced by the rigidness or fiexibility of the polymer mainchains. (Calculation of the $N$ number is described in the Results and Discussion Section. It is entirely the same as described in our first paper. ${ }^{1}$ ) Thus the rigid carbon-carbon double bond in 1 gave smaller $N$ numbers when compared with the flexible (five methylenes) main-chain in 2. ${ }^{1}$ An introduction of tere- 
phthalic acid unit instead of an aliphatic diacid unit in $\mathbf{3}$ also gave a decreased $N$ number. Accumulative substitutions of bulky phenyl and methyl groups in $\mathbf{4}$ greatly decreased its extent of side-chain crystallization.

In this paper, 1-alkynes with even carbon numbers from 6 to 18 were polymerized with transition metal catalysts, and the poly(1alkynes) were studied as a type of comb-like polymer. The conjugated polyene main-chain of poly(1-alkenes) was considered as rigid when compared with the polymethylene main-chain of poly(1-alkenes). Differential scanning calorimetry (DSC) study on the extent of their side-chain crystallization revealed as expected that poly(1-alkynes) give smaller $N$ numbers than poly(1-alkenes).

\section{EXPERIMENTAL}

\section{Reagents}

All 1-alkyne monomers (1-hexyne, 1-octyne, 1-decyne, 1-dodecyne, 1-tetradecyne, 1-hexadecyne, and 1-octadecyne) were of guaranteed grade obtained from Tokyo Kasei (Tokyo), and distilled over calcium hydride. Tungsten(VI) chloride, molybdenum(V) chloride, iron(III) acetylacetonate, triethylaluminum, and tetraphenyltin were also of guaranteed grade and used as received. Methyl benzoate and dioxane were refluxed over sodium and distilled. Other solvents were purified as usual.

\section{Polymerization}

A typical procedure of the polymerization experiment of 1-alkyne monomers is as follows. In a test tube, a mixture of tungsten(VI) chloride (catalyst, $474 \mathrm{mg}, 0.09 \mathrm{mmol}$ ) and tetraphenyltin (cocatalyst, $435 \mathrm{mg}, 0.09 \mathrm{mmol}$ ) in dioxane (solvent, $4.5 \mathrm{ml}$ ) was taken and aged for $15 \mathrm{~min}$ at $303 \mathrm{~K}$. Then 1-octadecyne (monomer, $1.6 \mathrm{ml}, 9.0 \mathrm{mmol}$ ) was added and polymerized for 1 day at $303 \mathrm{~K}$. A white precipitate was recovered by pouring the mixture into a large volume of methanol, and reprecipitated three times by using chloro- form-methanol. Then this product was stirred in ethanol at $333 \mathrm{~K}$ for $10 \mathrm{~min}$ and the supernatant solution was discarded while hot. This hot ethanol-extraction was repeated three times and the residual poly(1-octadecyne) was dried under vacuum. Other combinations of a monomer, catalyst, cocatalyst, and solvent were given in Table I. Work-up procedures were the same as above.

\section{Measurements}

Infrared (IR) spectra were recorded for $\mathrm{KBr}$ discs on a Jasco IR Report 100 spectrometer. ${ }^{1} \mathrm{H}(200 \mathrm{MHz})$ and ${ }^{13} \mathrm{C}(50 \mathrm{MHz})$ nuclear magnetic resonance (NMR) spectra were recorded on a Varian XL-200 spectrometer for $5-10 \mathrm{w} / \mathrm{v} \%$ sample solutions. Molecular weight determination of polymers was carried out by using a Tosoh HLC 803D gel permeation chromatograph with GMX-, G1000-, G2000-, and G4000-HXL poly(styrene) gel columns in series. The eluent was tetrahydrofuran and its flow rate was $1 \mathrm{ml} \mathrm{min}^{-1}$ at $313 \mathrm{~K}$. The retention time for each sample was read at the peak top and assumed as the number-average molecular weight $\left(M_{n}\right)$ with reference to poly(styrene) standards. Thermal analysis of polymers was carried out by using a Seiko I TA station with a DSC unit. Each sample of $c a$. $5 \mathrm{mg}$ was taken in an aluminum pan with lid and recorded its heating and cooling trace repeatedly by normalizing to a common weight $(10 \mathrm{mg})$. Heating and cooling rates were $10 \mathrm{~K} \mathrm{~min}^{-1}$. The heat capacity of this apparatus was calibrated by the following standards and averaged: benzene $(\mathrm{mp} 278.2 \mathrm{~K})$, benzophenone $(321.4 \mathrm{~K})$, indium $(429.8 \mathrm{~K})$, and tin $(505.1 \mathrm{~K})$. Gas chromatography analysis was carried out on a Shimazu GC-4C gas chromatograph with a $1 \mathrm{~m}$ Silicone E-301 column at $353 \mathrm{~K}$. The carrier gas was hydrogen and its flow rate was $50 \mathrm{ml} \mathrm{min}^{-1}$. X-Ray diffraction patterns were recorded for powder samples using $\mathrm{Ni}$-filtered $\mathrm{Cu}-K_{\alpha}$ radiation on a Rigaku-Denki RAD II refractometer. 


\section{RESULTS AND DISCUSSION}

\section{Polymerization}

A large number of papers has been published on the polymerization of substituted acetylenes as well as acetylene itself. Among them, Masuda and his coworkers' works ${ }^{5,6}$ should be referred to. They studied various transition metal catalysts, cocatalysts and solvents which polymerize substituted acetylenes to give polymers of very high molecular weight via a metathesis mechanism. In this study on the polymerization of 1-alkynes, we utilized their tungsten (W), molybdenum (Mo), and iron
(Fe) catalysts.

Table I summarizes the polymerization of 1-alkynes in this study. 1-Alkynes studied are 1-hexyne, 1-octyne, 1-decyne, 1-dodecyne, 1tetradecyne, 1-hexadecyne, and 1-octadecyne. The combinations of a catalyst, cocatalyst, and solvent are given.

The polymerization of alkynes is often accompanied by the formation of a cyclotrimer, trialkylbenzene. The contamination with such trialkylbenzenes might disturb the determination of the extents of crystallization of comb-like poly(1-alkynes) and therefore the crude products of polymerization were extract-

Table I. Polymerization of 1-alkynes ${ }^{\mathrm{a}}$

\begin{tabular}{|c|c|c|c|c|c|c|c|}
\hline \multirow{3}{*}{ Run } & \multirow{3}{*}{ 1-Alkyne } & \multirow{3}{*}{$\operatorname{mol~1}{ }^{-1}$} & \multirow{3}{*}{ Catalyst $^{b}$} & \multirow{3}{*}{ Solvent } & \multicolumn{3}{|c|}{ Polymer } \\
\hline & & & & & Yield $^{c}$ & & \\
\hline & & & & & $\%$ & & \\
\hline 1 & Hexyne & 0.8 & W & Toluene & 45 & 0.45 & 55 \\
\hline 2 & Hexyne & 1.0 & W & Toluene & 26 & 0.53 & 65 \\
\hline 3 & Hexyne & 1.0 & W & Dioxane & 59 & 1.5 & 182 \\
\hline 4 & Hexyne & 1.0 & Mo & $\mathrm{PhCOOMe}$ & 50 & 0.62 & 75 \\
\hline 5 & Hexyne & 2.0 & Mo & PhCOOMe & 46 & 1.1 & 134 \\
\hline 6 & Hexyne & 1.0 & $\mathrm{Fe}$ & Toluene & 32 & 0.57 & 69 \\
\hline 7 & Hexyne & 2.0 & $\mathrm{Fe}$ & Toluene & 15 & 0.87 & 105 \\
\hline 8 & Octyne & 1.0 & W & Dioxane & 68 & 2.7 & 245 \\
\hline 9 & Octyne & 2.0 & W & Dioxane & 67 & 3.3 & 299 \\
\hline 10 & Octyne & 2.0 & Mo & PhCOOMe & 34 & 0.61 & 56 \\
\hline 11 & Decyne & 2.0 & W & Toluene & 78 & 2.6 & 191 \\
\hline 12 & Dodecyne & 2.0 & W & Dioxane & 73 & 3.0 & 180 \\
\hline 13 & Dodecyne & 2.0 & Mo & PhCOOMe & 57 & 1.3 & 77 \\
\hline 14 & Tetradecyne & 0.5 & W & Toluene & 61 & 0.71 & 36 \\
\hline 15 & Tetradecyne & 0.7 & W & Toluene & 40 & 0.74 & 38 \\
\hline 16 & Tetradecyne & 1.0 & W & Toluene & 80 & 0.95 & 49 \\
\hline 17 & Tetradecyne & 1.0 & W & Toluene & 70 & 1.2 & 60 \\
\hline 18 & Tetradecyne & 1.6 & W & Toluene & 78 & 1.7 & 87 \\
\hline 19 & Tetradecyne & 2.2 & W & Toluene & 76 & 2.2 & 113 \\
\hline 20 & Tetradecyne & 1.0 & W & Dioxane & 73 & 1.4 & 72 \\
\hline 21 & Tetradecyne & 2.0 & W & Dioxane & 76 & 3.5 & 177 \\
\hline 22 & Tetradecyne & 2.0 & Mo & PhCOOMe & 55 & 1.1 & 56 \\
\hline 23 & Tetradecyne & 1.0 & $\mathrm{Fe}$ & Toluene & 70 & 1.3 & 67 \\
\hline 24 & Hexadecyne & 1.0 & W & Dioxane & 64 & 2.3 & 103 \\
\hline 25 & Octadecyne & 1.0 & W & Dioxane & 64 & 2.7 & 108 \\
\hline 26 & Octadecyne & 2.0 & W & Dioxane & 21 & 4.3 & 171 \\
\hline
\end{tabular}

a Polymerized at $303 \mathrm{~K}$ for $24 \mathrm{~h}$, with a $20 \mathrm{mmol}^{-1}$ catalyst concentration.

b Abbreviations of catalyst-cocatalyst combinations are: $\mathrm{W}=$ tungsten(VI) chloride-tetraphenyltin; $\mathrm{Mo}=$ molybdenum(V) chloride-tetraphenyltin; $\mathbf{F e}=$ iron(III) acetylacetonate-triethylaluminum.

c After hot ethanol extraction. 


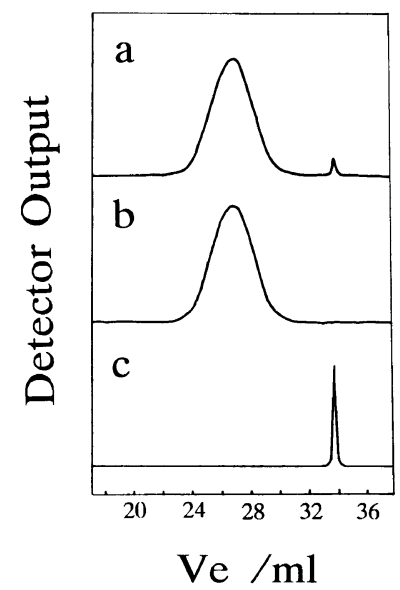

Figure 1. GPC traces of the product of polymerization of 1-hexyne: (a), before; (b), after the hot ethanol extraction; (c), tridecylbenzene.

ed with hot ethanol before isolating the poly (1-alkynes). Figure 1 shows the GPC traces of the product in the case of poly(1-dodecyne) (a) before and (b) after the extraction as well as of (c) an authentic sample of tridecylbenzene. The small peak at an elution volume $(\mathrm{Ve})$ of about $34 \mathrm{ml}$ in Figure 1a could be attributed to tridecylbenzenes and was completely removed by the extraction as shown in Figure 1b. The peak area of trialkylbenzene was found about $2 \%$ of the polymer peak area for all the 1-alkynes and catalyst species. Further study on the structure of tridecylbenzenes isolated from the hot ethanol-extracts through their gas chromatography, IR, ${ }^{1} \mathrm{H}$ and ${ }^{13} \mathrm{C}$ NMR experiments revealed that 1,2,4- and 1,3,5-isomers were formed in the ratio of $3: 7$. In Table I, the polymer yields are given for the product after the hot ethanol extraction. Loss of products due to lower molecular weight fractions as well as handling small quantity of samples might be involved.

In order to obtain poly(1-alkyne) samples of high molecular weight, various combinations of catalyst, cocatalyst, and solvent were utilized with reference to Masuda et al.'s works. Use of an oxygen-containing solvent such as dioxane or methyl benzoate, or a monomer feed in higher concentration led to enough high molecular weight samples as shown in Table I. The effect of molecular weight on the thermal behavior of poly(1-alkynes) will be discussed below.

At the same time, the combinations of catalyst, cocatalyst, and solvent, as well as monomer will affect the configuration of polymers. trans- and cis-Configurations are possible with respect to the main-chain carbon-carbon double bond in poly(1-alkynes).

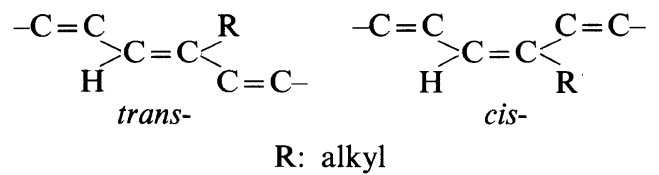

In the case of tert-butylacetylene, the $\mathrm{W}$ catalyst in hydrocarbon solvents has been reported to give a polymer of about the same amounts of trans- and cis-structures and the Mo catalyst in oxygen-containing solvents to give a polymer of exclusively cis-structure. ${ }^{7}$ Phenylacetylene generally gave trans-rich polymers by the $\mathrm{W}$ catalyst and cis-rich polymers by the Mo catalyst. ${ }^{8} \mathrm{~A}$ minor solvent effect was observed. In the case of 1-butyne, the W, Mo, and $\mathrm{Fe}$ catalysts gave constantly $80 \%$ cispolymers. ${ }^{9}$ Solvent effect was also negligible. It seems that the polymerization of alkynes with a branched and bulky substituent gives polymers of different configurations when polymerized by various metal catalysts whereas the polymerization of alkynes with $n$-alkyl substituents is not influenced by the catalyst used.

Poly(1-hexynes), obtained in this study, by using the $\mathrm{W}, \mathrm{Mo}$, and $\mathrm{Fe}$ catalysts are compared by their IR, ${ }^{1} \mathrm{H}$, and ${ }^{13} \mathrm{C}$ NMR spectra in Figures $2-4$ to study their configurations. These spectra are easily assigned to the poly(1-hexyne) structure in each case, but show only trivial difference with the catalyst used. Other higher poly(1-alkynes) in Table I showed almost the same spectra except for the varying peak intensities of $\mathrm{CH}_{2}$ absorptions. Spectral difference, if obvious, 


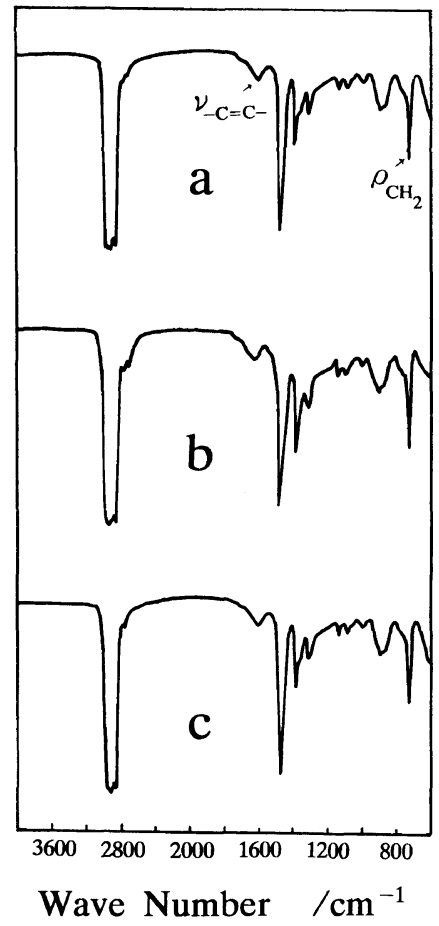

Figure 2. IR Spectra of poly(1-hexyne): (a), by $\mathrm{WCl}_{6}-$ $\mathrm{Sn}\left(\mathrm{C}_{6} \mathrm{H}_{5}\right)_{4}$ in toluene; (b), by $\mathrm{MoCl}_{5}-\mathrm{Sn}\left(\mathrm{C}_{6} \mathrm{H}_{5}\right)_{4}$ in methyl benzoate; (c), by $\mathrm{Fe}$ (III) acetylacetonate in toluene.

might have been ascribed to the difference in polymer configurations. At this point, we can not comment whether the poly(1-alkynes) obtained by the three catalysts are of a similar configuration or they do not show any configurational characteristics in the spectra.

\section{IR Spectra and X-Ray Diffraction Patterns}

Poly(1-alkynes) showed in their IR spectra a single absorption peak at $720 \mathrm{~cm}^{-1}$ in the $\mathrm{CH}_{2}$ rocking mode region. Chapman ${ }^{10}$ assigned such single peak at $720 \mathrm{~cm}^{-1}$ to the hexagonal-packed paraffin compounds and a doubly split peak at 727 and $719 \mathrm{~cm}^{-1}$ to the orthorhombic-packed paraffins. X-Ray diffraction patterns showed peaks at $2 \theta=21^{\circ}$ just as did other comb-like polymers. Thus the alkyl side-chains of poly(1-alkynes) are considered to crystallize in hexagonal packing as those of other many comb-like polymers. ${ }^{11}$

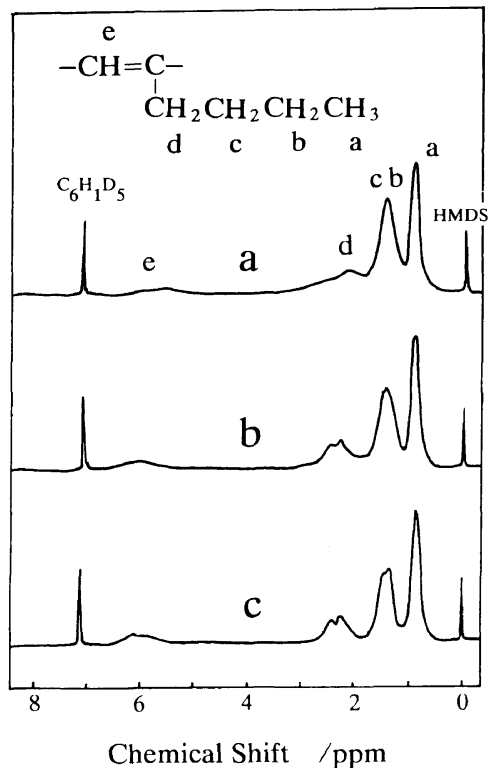

Figure 3. ${ }^{1} \mathrm{H}$ NMR Spectra of poly(1-hexyne): (a), (b), and (c) are referred to the catalyst, cocatalyst, and solvent as in Figure 2.

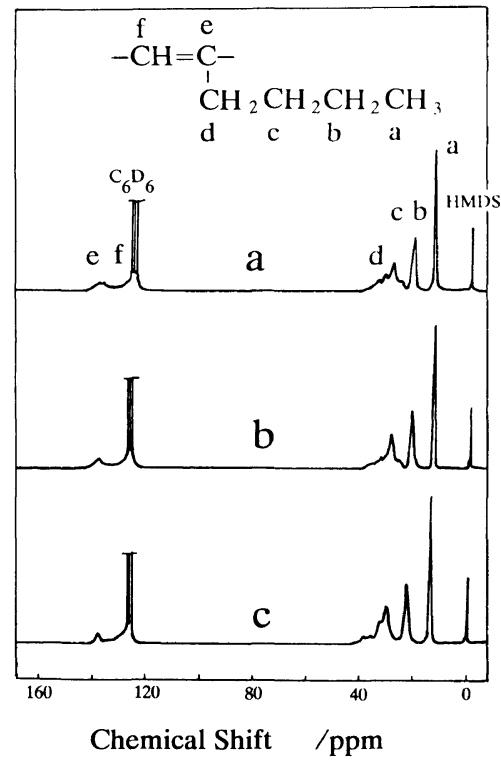

Figure 4. ${ }^{13} \mathrm{C}$ NMR Spectra of poly(1-hexyne): (a), (b), and (c) are referred to the catalyst, cocatalyst, and solvent as in Figure 2.

Thermal Analysis and the Extent of Crystallization

The side-chain crystallization of comb-like 

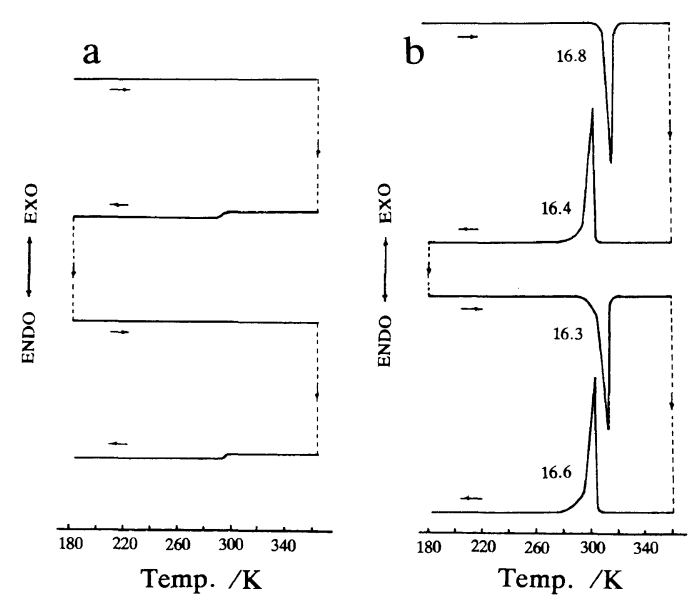

Figure 5. Repeated DSC traces of (a) poly(1-hexyne) and of (b) poly(1-octadecyne). Numerical values in (b) are referred to $\Delta H_{\mathrm{m}}$ or $\Delta H_{\mathrm{c}}$ given in $\mathrm{kJ}$ repeating unit ${ }^{-1} \mathrm{~mol}^{-1}$ calculated from the peak areas. poly(1-alkynes) was studied by using DSC in the same manner as for the other comb-like polymers 1-4 so far studied. Figure 5 shows typical repeated DSC traces of (a) poly(1hexyne) and of (b) poly(1-octadecyne). Poly(1hexyne) with a short $\mathrm{C}_{4}$-substituent shows, when cooling, clear inflection points at about $303 \mathrm{~K}$ which should be ascribed to its glass-transition temperature $\left(T_{\mathrm{g}}\right)$. Poly(1-octadecyne) with a long $\mathrm{C}_{16}$-substituent shows, when heating and cooling, sharp endothermic and exothermic peaks at $303-313 \mathrm{~K}$ which should be ascribed to its melting and crystallizing temperature $\left(T_{\mathrm{m}}\right.$ and $\left.T_{\mathrm{c}}\right)$, respectively. The peak areas are, when repeated, almost the same as given in the heat of melting and crystallization in Figure 5.

Table II. Thermal analysis of poly(1-alkynes) ${ }^{\mathbf{a}}$

\begin{tabular}{|c|c|c|c|c|c|c|c|}
\hline \multirow{2}{*}{ Run } & \multirow{2}{*}{ Poly(1-alkyne) } & \multirow{2}{*}{$\mathrm{C}_{n}$} & \multirow{2}{*}{ DP } & \multirow{2}{*}{$\frac{T_{\mathbf{g}}}{\mathbf{K}}$} & \multirow{2}{*}{$\begin{array}{l}T_{\mathrm{c}} \\
\mathrm{K}\end{array}$} & \multirow{2}{*}{\multicolumn{2}{|c|}{ 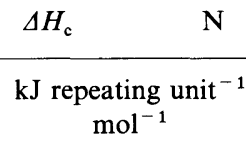 }} \\
\hline & & & & & & & \\
\hline 1 & Poly(1-hexyne) & 4 & 55 & 302.5 & & & \\
\hline 2 & Poly(1-hexyne) & 4 & 65 & 302.5 & & & \\
\hline 3 & Poly(1-hexyne) & 4 & 182 & 293.7 & & & \\
\hline 4 & Poly(1-hexyne) & 4 & 75 & 302.5 & & & \\
\hline 5 & Poly(1-hexyne) & 4 & 134 & 289.3 & & & \\
\hline 6 & Poly(1-hexyne) & 4 & 69 & 302.5 & & & \\
\hline 7 & Poly(1-hexyne) & 4 & 105 & 302.5 & & & \\
\hline 8 & Poly(1-octyne) & 6 & 245 & 296.4 & & & \\
\hline 9 & Poly(1-octyne) & 6 & 299 & 304.0 & & & \\
\hline 10 & Poly(1-octyne) & 6 & 56 & 294.2 & & & \\
\hline 11 & Poly(1-decyne) & 8 & 191 & 305.5 & & & \\
\hline 12 & Poly(1-dodecyne) & 10 & 180 & & 235.4 & 1.6 & 0.5 \\
\hline 13 & Poly(1-dodecyne) & 10 & 77 & & 227.3 & 1.7 & 0.5 \\
\hline 14 & Poly(1-tetradecyne) & 12 & 36 & & 248.6 & 8.4 & 2.7 \\
\hline 15 & Poly(1-tetradecyne) & 12 & 38 & & 248.8 & 8.4 & 2.6 \\
\hline 16 & Poly(1-tetradecyne) & 12 & 49 & & 256.2 & 7.5 & 2.4 \\
\hline 17 & Poly(1-tetradecyne) & 12 & 60 & & 256.4 & 7.7 & 2.5 \\
\hline 18 & Poly(1-tetradecyne) & 12 & 87 & & 265.4 & 6.3 & 2.1 \\
\hline 19 & Poly(1-tetradecyne) & 12 & 113 & & 265.2 & 6.4 & 2.1 \\
\hline 20 & Poly(1-tetradecyne) & 12 & 72 & & 265.7 & 6.4 & 2.1 \\
\hline 21 & Poly(1-tetradecyne) & 12 & 177 & & 264.6 & 6.1 & 2.0 \\
\hline 22 & Poly(1-tetradecyne) & 12 & 56 & & 262.1 & 6.0 & 2.0 \\
\hline 23 & Poly(1-tetradecyne) & 12 & 67 & & 262.7 & 6.4 & 2.1 \\
\hline 24 & Poly(1-hexadecyne) & 14 & 103 & & 289.4 & 12.3 & 4.0 \\
\hline 25 & Poly(1-octadecyne) & 16 & 108 & & 306.4 & 20.7 & 6.7 \\
\hline 26 & Poly(1-octadecyne) & 16 & 171 & & 305.2 & 20.0 & 6.5 \\
\hline
\end{tabular}

\footnotetext{
a Run number is identical to that in Table I, respectively.
} 
Next, the effect of molecular weight of a polymer on its thermal behavior was studied. In Table I, runs 14-21 show the polymerization of 1-tetradecyne by the $\mathrm{W}$ catalyst under various conditions giving poly(1-tetradecyne) samples of the molecular weight ranging from $0.71 \times 10^{4}$ to $3.5 \times 10^{4}$. All the poly(1-tetradecyne) samples showed sharp $T_{\mathrm{m}}$ and $T_{\mathrm{c}}$ peaks in their repeated DSC traces as the above poly(1-octadecyne). The $T_{\mathrm{c}}$ value increased with the degree of polymerization (DP) of a polymer sample as shown in Table II. From the first cooling $T_{\mathrm{c}}$ peak area, the heat of crystallization $\left(\Delta H_{\mathrm{c}}\right)$ of the side-chains of a polymer was determined. Then the $\Delta H_{\mathrm{c}}$ values were transformed into the $N$ numbers by dividing $\Delta H_{\mathrm{c}}$ by a reported heat of crystallization for hexagonal-packed $n$-paraffins $(3.08$ $\left.\mathrm{kJCH}_{2}{ }^{-1} \mathrm{~mol}^{-1}\right){ }^{12}$ The $\Delta H_{\mathrm{c}}$ and the $N$ numbers thus obtained are given in Table II for all poly(1-alkynes) as well as poly(1-tetradecynes). Figure 6 plots the $N$ number for poly(1-tetradecynes) against the DP of that sample and shows that the $N$ number turns out constant above $\mathrm{DP}=50$. Each of the other poly(1-alkynes) in Table II has a high DP over this threshold DP and hence its thermal behavior can be considered as intrinsic to that polymer, not affected by its molecular weight.

It seems curious and hardly be explained that the poly(1-tetradecynes) of lower DP than the threshold DP gave larger $N$ numbers. This has never been observed for other various types of comb-like polymers we studied. The phenome-

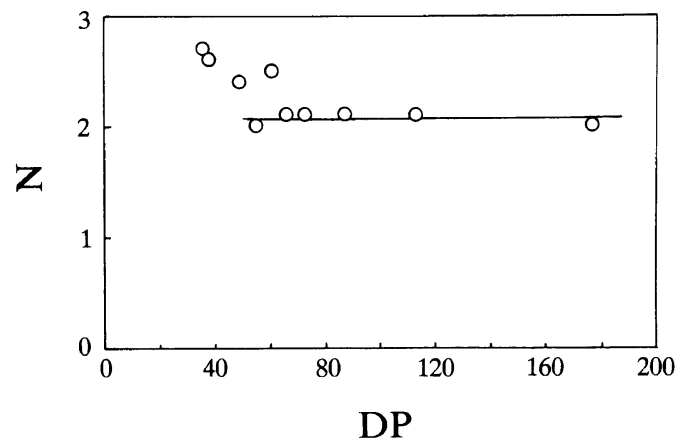

Figure 6. $N$ numbers against DP of poly(1-tetradecynes). non may be related to the fact that paraffins of medium molecular weight are well crystallizing.

Table II also shows that the catalyst, W, Mo, or Fe, has little effect on the $\Delta H_{\mathrm{c}}$ or the $N$ numbers of poly(1-alkynes). The catalyst effect on the poly(1-alkyne) configuration is not clear as described above. Now it is found that the $\Delta H_{\mathrm{c}}$ or the $N$ numbers do not depend on the catalyst. It should be noticed that the configuration of some comb-like polymers has little effect on the extent of their side-chain crystallization. ${ }^{11}$

Figure 7 collects the typical cooling DSC traces of poly(1-alkynes) with $\mathrm{C}_{4}-\mathrm{C}_{16}$ sidechains. Poly(1-hexyne), poly(1-octyne), and poly(1-decyne) with short $\mathrm{C}_{4}, \mathrm{C}_{6}$, and $\mathrm{C}_{8}$ side-chains show only $T_{\mathrm{g}}$ inflection whereas poly(1-dodecyne), poly(1-tetradecyne), poly(1hexadecyne), and poly(1-octadecyne) with long $\mathrm{C}_{10}-\mathrm{C}_{16}$ side-chains show $T_{\mathrm{m}}$ peaks. This is in accordance with the general behavior of comb-like polymers with various side-chain lengths that the side-chain crystallization of a comb-like polymer occurs when its side-chain contains more than $8-10$ carbons. ${ }^{11}$ The fact that when polymers with short side-chains are amorphous whereas the same polymers with long side-chains are crystalline is considered to indicate that crystallizing are the side-chains in the latter polymers.

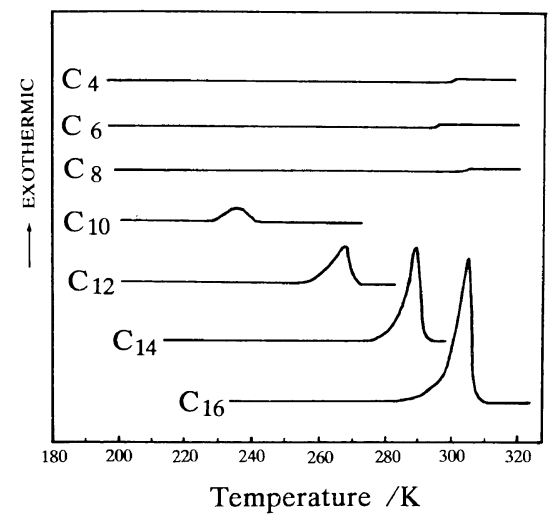

Figure 7. Typical cooling DSC traces of poly(1-alkynes). $\mathrm{C}_{n}$ designates the carbon number in the alkyl side-chain. 


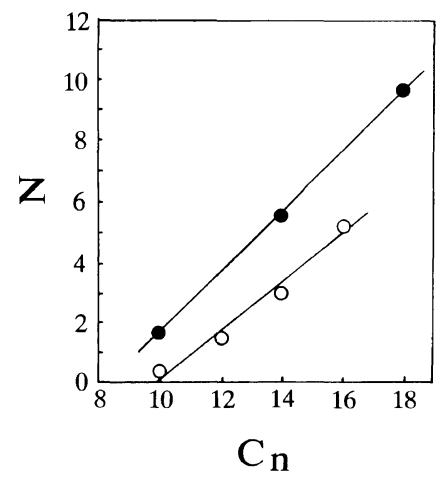

Figure 8. $\quad N$ Numbers of poly(1-alkynes) $(O)$ and poly(1-alkenes) (O). $\mathrm{C}_{n}$ designates the carbon number in the alkyl side-chain.

Table III. $T_{\mathrm{g}}$ 's of relevant polymers ${ }^{14}$

\begin{tabular}{|c|c|c|}
\hline \multirow[b]{2}{*}{$\mathrm{R}$} & \multicolumn{2}{|c|}{$T_{\mathrm{g}} / \mathrm{K}$} \\
\hline & $\begin{array}{c}-\mathrm{C}-\mathrm{C}- \\
\mathrm{I} \\
\mathrm{R}\end{array}$ & $-\mathrm{C}=\underset{\mathrm{l}}{\mathrm{C}-}$ \\
\hline$n-\mathrm{C}_{4} \mathrm{H}_{9}-$ & 223 & $300^{\mathrm{a}}$ \\
\hline$n-\mathrm{C}_{6} \mathrm{H}_{13}-$ & 208 & $295^{a}$ \\
\hline $\mathrm{C}_{6} \mathrm{H}_{5}$ & 373 & 393 \\
\hline
\end{tabular}

a Present study.

The $N$ numbers of poly(1-alkynes) are plotted in Figure 8 against the carbon numbers .$\left(\mathrm{C}_{n}\right)$ of their alkyl side-chains. Figure 8 also plots the $N$ numbers of atactic poly(1-alkenes) calculated from the literature data. ${ }^{13}$ Both comb-like polymers show the increasing $N$ numbers with the increasing side-chain carbon numbers. The poly(1-alkyne), however, shows a constantly smaller $N$ number (about $2 \mathrm{CH}_{2}$ units) than the poly(1-alkene) of the same side-chain carbon number. This result should come from the rigidness or flexibility of the polymer main-chain. The rigid conjugated polyene main-chain structure of poly(1alkynes) resulted in the lower extent of side-chain crystallization than the flexible polymethylene main-chain structure of poly(1alkenes). Table III collects the $T_{\mathrm{g}}$ of some relevant polymers and demonstrates the difference in rigidness or flexibility between polyene and polymethylene main-chain structures. Each of the substituted poly(alkynes) shows a higher $T_{\mathrm{g}}$ and hence a more rigid main-chain structure than the corresponding polyalkene. In conclusion, this paper gave a new example that the extent of the side-chain crystallization of comb-like polymers is influenced by the rigidness or flexibility of the polymer main-chain.

\section{REFERENCES}

1. K. Yokota, T. Kougo, and T. Hirabayashi, Polym. J., 15, 891 (1983).

2. K. Yokota and T. Hirabayashi, Polym. J., 18, 177 (1986).

3. T. Hirabayashi, K. Kasabou, and K. Yokota, Polym. J., 20, 911 (1988).

4. T. Hirabayashi, T. Kikuta, K. Kasabou, and K. Yokota, Polym. J., 20, 693 (1988).

5. T. Masuda and T. Higashimura, Acc. Chem. Res., 17, 51 (1984).

6. T. Masuda and T. Higashimura, Adv. Polym. Sci., 81, 121 (1986).

7. Y. Okano, T. Masuda, and T. Higashimura, Polym. J., 14, 477 (1982).

8. T. Masuda, N. Sasaki, and T. Higashimura, Macromolecules, 8, 717 (1975).

9. T. Masuda, Y. Okano, K. Tamura, and T. Higashimura, Polymer, 26, 793 (1985).

10. D. Chapman, J. Chem. Soc., 4489 (1957).

11. N. A. Plate and V. P. Shibaev, Ed., "Comb-Shaped Polymers and Liquid Crystals," Plenum Press, New York, N.Y., 1987, Chapter 1.

12. M. G. Broadhurst, J. Res. Natl. Bur. Stand., 66A, 241 (1962).

13. P. L. Maganini, D. Lupinacci, F. Cotrozzi, and A. Andruzzi, Makromol. Chem., Rapid Commun., 1, 557 (1980).

14. J. Brandrup and E. H. Immergut, Ed., "Polymer Handbook," 3rd ed, Wiley-Interscience, New York, N.Y., 1989, p VI-209. 